\title{
REVIEW
}

\section{Central Neuropathic Mechanisms in Pain Signaling Pathways: Current Evidence and Recommendations}

\author{
Omar Viswanath · Ivan Urits · James Burns · Karina Charipova • \\ Kyle Gress · Alexandra McNally • Richard D. Urman · Ali Welschmeyer • \\ Amnon A. Berger · Hisham Kassem • Manuel G. Sanchez • Alan D. Kaye • \\ Treniece N. Eubanks · Elyse M. Cornett · Anh L. Ngo
}

Received: February 12, 2020 / Published online: April 10, 2020

(C) The Author(s) 2020

\section{ABSTRACT}

Purpose: This is a comprehensive review of the current literature on central neuropathic pain mechanisms that is secondary to spinal cord injury. It reviews recent and seminal findings on the pathophysiology, diagnosis, and treatment and compares treatment options and recommendations.

Recent Findings: Neuropathic pain (NP) is a common complication of spinal cord injury (SCI). Chronicity of NP is attributed to increased

Enhanced Digital Features To view enhanced digital features for this article go to https://doi.org/10.6084/ m9.figshare.12058299.

O. Viswanath

Valley Anesthesiology and Pain

Consultants-Envision Physician Services, Phoenix, AZ, USA

O. Viswanath

Department of Anesthesiology, University of Arizona College of Medicine Phoenix, Phoenix, AZ, USA

O. Viswanath

Department of Anesthesiology, Creighton

University School of Medicine, Omaha, NE, USA

I. Urits $(\bowtie) \cdot$ A. A. Berger

Beth Israel Deaconess Medical Center, Department of Anesthesiology, Critical Care, and Pain Medicine, Harvard Medical School, Boston, MA, USA

e-mail: ivanurits@gmail.com abundance of inflammatory mediators and ion channel dysfunction leading to afferent nerve sensitization; nerve damage and nerve-glia cross talk have also been implicated. Conventional treatment is medical and has had limited success. Recent studies have made headway in identifying novel biomarkers, including microRNA and psychosocial attributes that can predict progress from SCI to chronic NP (CNP). Recent advances have provided evidence of efficacy for two promising drugs. Baclofen was able to provide good, long-lasting pain relief. Ziconotide, a voltage-gated calcium channel blocker, was studied in a small trial and was able to provide good analgesia in most participants. However, several participants had to be

J. Burns · K. Charipova - K. Gress · A. McNally ·

A. Welschmeyer

Georgetown University School of Medicine,

Washington, DC, USA

\section{R. D. Urman}

Department of Anesthesiology, Perioperative and Pain Medicine, Brigham and Women's Hospital, Boston, MA, USA

\section{H. Kassem}

Department of Anesthesiology, Mount Sinai Medical Center, Miami Beach, FL, USA

M. G. Sanchez · A. L. Ngo

Department of Pain Medicine, Pain Specialty Group, Newington, $\mathrm{NH}$, USA 
withdrawn because of worrisome creatine phosphokinase (CPK) elevations, and further studies are required to define its safety profile. Non-medical interventions include brain sensitization and biofeedback techniques. These methods have recently had encouraging results, albeit preliminary. Case reports of non-conventional techniques, such as hypnosis, were also reported.

Summary: CNP is a common complication of SCI and is a prevalent disorder with significant morbidity and disability. Conventional medical treatment is limited in efficacy. Recent studies identified baclofen and ziconotide as possible new therapies, alongside non-medical interventions. Further research into the pathophysiology is required to identify further therapy candidates. A multidisciplinary approach, including psychosocial support, medical and non-medical interventions, is likely needed to achieve therapeutic effects in this difficult to treat syndrome.

Keywords: Allodynia; Baclofen; Central neuropathic pain; Chronic pain; Spinal cord injury; Ziconotide

\section{Key Summary Points}

Central neuropathic pain (CNP) is a common complication of spinal cord injury (SCI) and is a prevalent disorder with significant morbidity and disability.

Conventional medical treatment is limited in efficacy but recent studies identified baclofen and ziconotide as possible new therapies, alongside non-medical interventions.

Further research into the pathophysiology is required to identify further therapy candidates.

A. D. Kaye - T. N. Eubanks - E. M. Cornett Department of Anesthesiology, Louisiana State University Health Shreveport, Shreveport, LA, USA

A. L. Ngo

Harvard Medical School, Boston, USA
A multidisciplinary approach, including psychosocial support, medical and nonmedical interventions, is likely needed to achieve therapeutic effects in this difficult to treat syndrome.

\section{INTRODUCTION}

Neuropathic pain is a common complication of spinal cord injury (SCI). It is defined as "pain caused by a lesion or disease of the somatosensory system" [1]. Symptoms include allodynia (pain due to an innocuous stimulus) and spontaneous shooting or burning pain associated with neuronal dysfunction [2]. The most common causes of SCI include motor vehicle accidents, falls, and acts of violence.

The etiology of chronic neuropathic pain (CNP) is complex and multidimensional. Neuroimmune interactions throughout the nervous system result in abnormal sensory signaling in the periphery, spinal cord (dorsal horn), and brain (thalamus and cortex) leading to the observed painful symptoms [3]. Increases in inflammatory mediators cause afferent nerve sensitization through altered expression of voltage-gated sodium, calcium, and potassium channels resulting in spontaneous pain without painful stimulation [3-5].

Macrophages, T lymphocytes, cytokines, and chemokines mediate the inflammatory responses that cause maladaptive nociceptor overstimulation [6]. Patients with CNP have up to three times higher levels of pro-inflammatory interleukin (IL)-2 and tumor necrosis factor-alpha (TNF $\alpha$ ) than those without pain [7]. MicroRNAs (miRNA) also influence CNP manifestations by regulating pain recognition proteins, promoting neurodegenerating inflammation, and stimulating Toll-like receptors in dorsal root ganglion sensory neurons (DRG) [6, 8-10].

Neuron-glial cell interactions are implicated in the pathogenesis of neuropathic pain. The activation of microglia and astrocytes in the spinal cord cause chemokine upregulation and increased signaling between neurons and glia 
[11]. Chemokines sensitize peripheral and central nerves following the nerve and tissue damage seen in SCI [12].

Current treatments for neuropathic pain show limited efficacy. The Special Interest Group on Neuropathic Pain suggest gabapentinoids, tricyclic antidepressants (TCAs), and selective serotonin-norepinephrine reuptake inhibitors (SNRI) as first-line treatments [13]. Lidocaine, caspaicin, tramadol, and opioids are second- and third-line treatments [13]. Limited symptom control suggests the need for novel therapeutic approaches.

Recent studies have investigated Botulinum toxin A (BTX), an inhibitory neurotoxin, as a potential future treatment for CNP. Preliminary studies, however, yield mixed results, which suggests further research is necessary. The objective of this manuscript is to review recent advances in the understanding of SCI and CNP.

This article is based on previously conducted studies and does not contain any studies with human participants or animals performed by any of the authors.

\section{EPIDEMIOLOGY}

An estimate upwards of $80 \%$ of patients following SCI may develop CNP, and the symptom progression of CNP secondary to SCI has been studied in depth. A meta-analysis of the symptom transition period (1-6 months and 1-12 months post-injury) showed that in patients with initial pain 1 month after injury, $72 \%$ had persistent symptoms at 6 months post-injury and $69 \%$ had persistent pain at 12 months postinjury [14]. Regression analysis showed that older age increased the likelihood of developing neuropathic pain [14]. A recent prospective study assessing predictors of pain following SCI showed that neuropathic pain increased with time while musculoskeletal pain decreased [15]. This study further suggested that early-onset sensory hypersensitivity, or allodynia, may predict later development of neuropathic pain [15].

Studies of long-term outcomes in the management of CNP show that patients with CNP are less likely to achieve clinically notable improvements in pain relief and function than those with peripheral neuropathic pain [16].

SCI-related neuropathic pain is associated with a lower response to placebo compared to other neuropathic pain conditions (e.g., HIV neuropathy) [17]. Direct damage to the spinal cord disrupts the physiologic mechanisms required to achieve normal placebo analgesic response [18].

Risk of developing CNP in SCI can be predicted with almost $90 \%$ accuracy on the basis of characteristic electroencephalography (EEG) markers of pain which appear prior to onset of physical pain [19]. Characteristic EEG findings in patients with current CNP or those who later develop CNP in SCI include reduced alpha band and absent theta and beta band in reaction to eye opening [20].

Several biomarkers have been used to identify neuropathic pain in patients with SCI. Specific genes known as differentially expressed genes, and miRNAs, such as mir-204-5p, mir519-3p, mir-20b-5p, and mir-6838-5p, have been identified and may serve as predictors of neuropathic pain [21]. These biomarkers may be potential therapeutic targets for prevention and treatment of neuropathic pain in the future [21].

Psychological stressors have been linked to neuropathic pain development in SCI. A 2018 cross-sectional study demonstrated that individuals who sustain SCI with increased psychological distress have increased propensity to develop CNP compared to SCI controls [22]. These psychological distresses include posttraumatic stress disorder (PTSD), anxiety, stress, depression, and pain catastrophizing [22].

\section{CLINICAL PRESENTATION AND DIAGNOSIS}

Patients with neuropathic pain present clinically with variable symptoms often including burning, tingling, and allodynia [2]. Several molecular mechanisms of nerve injury have been proposed to explain these symptoms. Alterations in long non-coding RNAs (lncRNAs) expression following SCI and peripheral nerve 
injury contribute to the pathophysiology of CNP [23]. Differentially expressed lncRNAs alter Toll-like receptors, calcium signaling, and peroxisome proliferator-activated receptor signaling causing CNP symptoms [24]. Spinal cord sensitization is due to reduced expression of Kcna2 antisense RNA, resulting in net decreased inhibitory signaling [25]. Differentially expressed lncRNAs disrupt normal ion channel signaling, signal transduction pathways, and inflammatory-mediated signaling in nociceptive neurons [25-27].

MicroRNA (miRNAs) dysregulation is commonly seen in SCI and might be a potential treatment target [28]. Increased inflammation, oxidative stress, and apoptosis associated with SCI are linked to changes in miRNA expression following injury [29]. MiRNAs are a promising target for future treatments which may aim to prevent aberrant miRNA expression or prevent downstream effects of deleterious miRNA pathways involved in neuropathic pain [30]. Current use of miRNA-targeted therapy is limited and warrants further research as a possible effective treatment.

Diagnosis of neuropathic pain is complex and rarely definitive. It is diagnosed using criteria established by Analgesic, Anesthetic, and Addiction Clinical Trial Translations, Innovations, Opportunities, and Networks public-private partnership and the American Pain Society, the Analgesic, Anesthetic, and Addiction Clinical Trial Translations, Innovations, Opportunities, and Networks-American Pain Society Pain Taxonomy (AAPT). Major diagnostic conditions include central pain associated with SCI, multiple sclerosis (MS), and central post-stroke pain (CPSP) [31]. AAPT diagnostic criteria consider common features, common comorbidities, pain consequences, risk factors, and neurobiologic and psychosocial mechanisms of patients affected with central pain [31]. The International Association for the Study of Pain criteria require history of nervous system lesion with a distribution of pain and sensory changes that are neuroanatomically plausible given the location of the lesion [31]. Confirmatory imaging (e.g., computed tomography, magnetic resonance imaging), neurophysiological, or biological tests should be used to confirm any history of SCI, stroke, MS, or other CNS lesions [31]. Despite fulfilling these criteria, the proposed diagnostic tools cannot provide an absolute diagnosis of neuropathic pain with certainty, and the possibility of other conditions should not be ruled out. Many patients with neuropathic pain symptoms also have one or more chronic overlapping pain conditions, further complicating definitive diagnosis $[31,32]$.

\section{PATHOPHYSIOLOGY AND PROPOSED MECHANISMS}

\section{Changes in Neuronal Cells Following SCI}

Following ischemic injury to the spinal cord dorsal horn, microglia cells are overstimulated $[33,34]$. Microglia cells in turn hyperproduce pro-inflammatory markers such as brain-derived neurotrophic factor (BDNF), chemokines, and cytokines, causing increased numbers of microglia cells, and excess neuronal excitability. A test group of rats underwent induced hind limb ischemia from O-ring use for $3 \mathrm{~h}$. Fourteen days following reperfusion, the rats were examined and found to have increased microglia, higher levels of BDNF which potentiates excitability between neurons and C-fibers, and a high presence of colony-stimulating factor (CSF1R) [33, 35]. CSF1R is a signaling factor in the proliferation of microglia cells and is typically highest in the postnatal period and lowest in the adult brain when microglial proliferation is rare. These microglia cells express $\mathrm{P}_{2} \mathrm{X}_{4}$ receptors, which adenosine triphosphate binds to after being released from damaged neurons, and promotes $\mathrm{Ca}^{2+}$ influx, causing increased tactile allodynia [36]. Tang et al. examined microglia proliferation in the setting of CSFR1 inhibitor PLX5622 and found it to be suppressed, confirming that the increase in CSFR1 increases microglia proliferation. Du et al. found that neural stem cell transplantation into damaged spinal cord dampens the $\mathrm{P}_{2} \mathrm{X}_{4}$ activity, and in turn decreases pain reaction in the test group. These studies conclude that ischemic damage reactivates the CSFR1 signaling pathway, causing microglia accumulation, and $\mathrm{P}_{2} \mathrm{X}_{4}$ 
receptors are upregulated increasing $\mathrm{Ca}^{2+}$ influx into these microglia. These events lead to heightened production of inflammatory markers and BDNF, resulting in post-ischemic pain, evidenced by the change in behavior of the mice such as chewing their paws $[33,36]$.

A second study examined neuropathic pain in the setting of sciatic nerve ligation. Lee et al. examined the activity of macrophages following ischemic damage of the peripheral nerve, and found an increase in number and macrophage colony-stimulating factor (M-CSF) [37]. These macrophages were found to have enhanced release of inflammatory cytokines, and VEGF, causing a breakdown in the blood-brain barrier. They identified that though damage occurred in a peripheral nerve, the M-CSF stimulated CSFR1 in the central nervous system, causing the same changes in microglia cells discussed in the first study. Lee et al. similarly identified that PLX5622 decreased CSFR1 and microglia proliferation, but also decreased M-CSF and macrophage proliferation, and overall decreased neuropathic pain enhancement in both the central and peripheral nervous systems [37].

Chhaya et al. explored the effect of exercise on macrophage and microglia proliferation following damage to C5 spinal cord. They found that mice in the non-exercise group had increased microglia cell proliferation $(10.7 \%$ increase, $p=0.01$ ) present within the dorsal horn when compared with mice in the exercise group $(6.78 \%, p>0.05)$. They also found that macrophages present in the dorsal root ganglia of the non-exercise group versus the exercise group were not significantly different from one another. This study suggests some potential benefit of exercise in decreasing microglial celldriven neuropathic pain [34].

\section{Various Mechanisms of Pain Response Due to Changes in Neuronal Cells Following SCI}

GABAergic (GABA) neurotransmitters are the leading players in many neuropathic pain pathways. In the uninjured spinal cord, $\mathrm{GABA}_{\mathrm{A}}$ and glycine function on dorsal horn neurons to cause an inhibitory effect preventing their firing. A main subunit that is involved in this pathway is the alpha-2-delta- $1\left(\alpha_{2} \delta-1\right)$ subunit of voltage-gated calcium channels [38]. This functions in neuron excitation fast pathways for neurotransmitter release, and is dampened by GABA. Kusuyama et al. found that $\alpha_{2} \delta-1$ is increased in the dorsal horn of the spinal cord following SCI in rats 7-21 days following injury. They also identified that these rats had a decreased threshold for pain withdrawal compared to control. Subsequently, following administration of pregabalin, only tactile allodynia was decreased, though overall expression of $\alpha_{2} \delta-1$ was decreased $(p<0.05)$, suggesting that treatment of SCI with GABAergic agents will decrease overall neuropathic pain response [38].

Another important aspect of neuron excitability is the potassium chloride cotransporter (KCC2). The KCC2 cotransporter standardly causes an efflux of chloride ions and an influx of potassium ions in dorsal horn neurons, keeping ion concentrations appropriate for depolarization upon excitatory stimulation. When $\mathrm{GABA}_{\mathrm{A}}$ and glycine are released, they cause the KCC2 transporter to allow an influx of chloride ions into the neuron, causing hyperpolarization, and preventing the neuron from firing [39]. When SCI occurs, KCC2 is downregulated, causing $\mathrm{GABA}_{\mathrm{A}}$ and glycine to be ineffective and resulting in a loss of inhibition of the dorsal horn neurons, leading to spasticity and pain. A study was conducted on 32 rats with spinal cord hemisection. The rats were assessed for allodynia to mechanical and thermal stimuli, and found to have a decreased threshold for withdraw $(P<0.001)$ bilaterally [39]. At day 21 postoperatively, the rats were deemed stable to undergo pharmacologic treatment with (4-bromo-3,6-dimethoxybenzocyclobuten-1-yl)methylamine hydrobromide, TCB-2. TCB-2 causes a release of $5-\mathrm{HT}_{2 \mathrm{~A}}$. This study established that this pathway causes a G-protein-coupled increase in protein kinase C, which causes an upregulation of KCC2 in the dorsal horn in SCI. Immunostaining for KCC2 before and after treatment with TCB-2 showed a $50 \%$ increase in KCC2 in the neuronal membrane $(P<0.0001)$ ipsilateral to the 
hemisection [39]. Post treatment with TCB-2 also found decreased allodynia to mechanical and thermal stimuli, further confirming its effectiveness in increasing KCC2 function and decreasing pain due to SCI.

A second study suggests a possible treatment to potentiate the benefits of TCB-2. This study targets miRNA miR-15a/16, which are overexpressed in SCI, causing a downregulation of G-protein-coupled receptor kinase 2 (GRK2). When GRK2 is decreased, allodynia is increased through the pathway discussed above, so research into preventing overexpression of miR$15 \mathrm{a} / 16$ can also improve SCI neuropathic pain [40]. Conversely, Yang et al. found that miR-128 was downregulated in microglia following SCI, and causing increased amounts of inflammatory subunits. This occurred by BDNF activating the p53 MAPK pathway, leading to a decrease in inhibition of inflammatory cytokines such as IL-6, IL-1B, and TNF $\alpha$, all of which are vital in causing neuropathic pain, suggesting it would be beneficial to explore this mechanism as a route to treating pain following SCI [41].

Miranpuri et al. explored the actual activity of these inflammatory markers, and found that the MAPK pathway activates matrix metalloproteinase 2 and 9, which cleave the inflammatory markers into their active forms, further potentiating neuropathic pain as a result [42]. Gui et al. utilized this information and studied bexarotent in the setting of SCI. They found that treatment with this drug inhibited the MAPK pathway, decreased microglia proliferation, and in turn decreased the activity of inflammatory markers $(p<0.001)$ compared with control [43]. Castany et al. treated mice 28 days post SCI with MR-309, which is a $\sigma 1 \mathrm{R}$ antagonist. This also inhibits MAPK and resulted in a decrease in inflammatory markers and mechanical and thermal allodynia in the injured mice $(p=0.022)$, showing another useful treatment of SCI neuropathic pain [44]. Interestingly, Sanna et al. explored the effects of lavender essential oils in neuropathic pain control. They studied mice with SCI and found that $100 \mathrm{mg} / \mathrm{kg}$ of essential oils hyperphosphorylated the MAPK pathway, preventing overactivation of the inflammatory pathway, and effectively decreased allodynia [45].
Liu et al. explored an additional inflammatory pathway in mice with SCI. They found an increase in CXCL12/CXCR4 in dorsal horn neurons. They then injected healthy mice intrathecally with these peptides and found that it resulted in hyperalgesia. Finally, they utilized neutralizing antibodies to CXCL12/CXCR4 in the mice with SCI and found an attenuation of the neuropathic pain. They suggest that this pathway contributes to overactivation of microglia, but it is still not exactly clear what the mechanism leading to hyperalgesia is [46]. Interestingly, a similar experiment found that CXCL9 and CXCL11 are also increased following SCI, but they do not cause hyperalgesia when injected intrathecally, suggesting that though they are upregulated they are not involved in the post-injury neuropathic pain response [47].

An important comment on the current lack of neuropathic pain management is highlighted by the frequent use of morphine. Only one in three patients with central neuropathic pain experience up to a $50 \%$ decrease in pain with the current regimens. Neurons in the dorsal root ganglia, microglia, and astrocytes express Toll-like receptor 4 (TLR4), which is known to be an important factor in increasing neuropathic pain [48]. Ellis et al. found that while opioids moderate pain through the typical mu receptor pathways, they can enhance neuropathic pain by stimulating TLR4 receptors for up to 4 weeks after use [48]. Additional to this potential increase in pain reactions, there is of course the side effect profile of opioids that must not be overlooked when assessing the need for new treatment of neuropathic pain following SCI [49].

\section{CLINICAL EVIDENCE}

There are multiple hypotheses for the etiology of neuropathic pain originating in different parts of the central and peripheral nervous systems.

\section{Development}

A European meta-analysis looked at the development of neuropathic pain in the setting of a 
recent SCI [50]. Over 1000 individuals were screened with a European Multi-Center Study about Spinal Cord Injury (EMSCI) pain questionnaire 1 month after injury and had their pain status assessed at 6 months and 12 months [50]. Risk factors for developing neuropathic pain at any time point following SCI were advanced age and motor and sensory preservation following less severe injury [50]. At the initial visit $27 \%$ of patients had neuropathic pain; $23 \%$ of patients who initially screened negative developed pain at either the 6- or 12 -month follow-up [50]. Interestingly, 30\% of patients that initially presented with pain saw resolution over the course of the 12-month period [50]. Thus, approximately 35\% of initially screened patients with SCI had neuropathic pain at the 12-month checkpoint [50]. While the pathophysiology of delayed development of neuropathic pain is not well understood, it is likely that the delay reflects time required for neuroma development [50]. This study demonstrated the importance of longterm follow-up both in patients presenting with and those presenting without symptoms immediately after SCI [50].

Recent studies have shed light on neurological deficit associated with SCI results not only from direct tissue damage but also from the inflammatory response that develops in the weeks following injury [51]. The alpha 7 nicotinic acetylcholine receptor ( $\alpha 7-n A C h R)$ plays a central role in downregulating inflammatory responses [51]. The $\alpha 7-n A C h R$ is encoded by the CHRNA7 gene, which in some individuals is fused with a partial duplication of gene $F A M 7 A$ to create $C H R F A M 7 A$ [51]. Individuals with the del2bp gene variant polymorphism of CHRFAM7A encode a protein called $\operatorname{dup} \Delta \alpha 7$, which suppresses $\alpha 7-n A C h R$ function and induces a pro-inflammatory phenotype [51]. When $\alpha 7-n A C h R$ is suppressed, levels of proinflammatory cytokines such as TNF $\alpha$ are thought to increase [51]. Huang and colleagues analyzed how the presence of CHRFAM7A contributes to outcomes after SCI [51]. When levels of noradrenergic metabolites and circulating cytokines were measured in patients with SCI and compared to controls, TNF $\alpha$ levels were found to be three times higher in patients with the del2bp gene variant compared to no deletion genotypes 3 weeks after injury [51]. Noradrenergic metabolite levels were unchanged immediately after injury but significantly decreased in carriers of the deletion 3 weeks following injury compared to non-carriers [51]. Interestingly, numeric pain scores in patients with the deletion were significantly higher compared to those without the gene variant [51].

In continuing the molecular approach to the post-SCI inflammatory response, gap junction (GJ) channels, specifically connexin43 (Cx43), have been implicated in the development of neuropathic pain [52]. Cx43 is the most abundant connexin in mammals and is most commonly expressed in astrocytic glial cells of the central nervous system [52]. While neuropathic pain treatments have traditionally focused on neurons, new disease models suggesting that Cx43 expression increases after SCI are now shifting the focus to glial cells [52]. It is thought that following nerve injury, upregulation of GJs and $\mathrm{Cx} 43$ causes sensitization of glial cells to pain media such as ATP and other inflammatory cytokines, resulting in increased synaptic pain signaling [52]. Of note, the functionality of connexins also includes extracellular exchange via unpaired hemichannels [53]. These specific hemichannels have been targeted by short peptides called peptidomimetics that act on components of the connexin pathways and ultimately prevent hemichannel opening. In rat models, these peptidomimetics have proven successful in reducing neuroinflammation after SCI [53]. With increased specificity, hemichannels can be targeted without inhibition of GJs using peptidomimetics, directed antibodies, or non-peptide analogues of connexin mimetic peptides. Further investigation is required to better understand the mechanism by which Cx43 and its hemichannel are involved in the pathogenesis of neuropathic pain to pave the way for future therapeutic strategies [52].

\section{Sensitization}

A Danish research group explored the modulatory response of the body to neuropathic pain 
and the process of sensitization in the presence of SCI [54]. They compared patients with acute, subacute, and chronic SCI to healthy controls to determine whether or not sensitization above the lesion was comparable between the groups [54]. They also assessed whether or not use of analgesics results in a central sensitization effect [54]. While unmedicated, patients in the SCI group had reduced pressure and pain detection thresholds as measured through pressure algometry and repetitive pin prick compared to the control group [54]. This observation supports the phenomenon of central sensitization, which involves neurologic structures such as the anterior cingulate cortex (ACC), hippocampus, and amygdala but is generally poorly understood [55]. However, individual patients in the SCI group did not demonstrate an increase in pain and pressure sensitization compared to their own baselines [54]. The overall group also did not appear sensitized to pain or pressure when patients were sorted by their medication histories into low (non-opioid), moderate (non-opioid, weak opioid), and heavy (strong opioid) medicated subgroups [54].

\section{Treatment}

Various medications have been assessed for their potential to reduce the intensity of neuropathic pain of various etiologies. First-line pharmacologic interventions for treatment of neuropathic main include gabapentinoids, TCAs, selective serotonin reuptake inhibitors (SSRIs), and SNRIs [56]. Unfortunately, controversy exists regarding the efficacy of even these first-line agents. Studies have suggested that there may be lack of evidence regarding successful treatment with amitriptyline, but this conclusion must be balanced against the many patients who have achieved pain relief with this drug [57]. In a case report of a patient with central neuropathic pain secondary to a diffuse glioma and refractory to these medications, the patient subsequently achieved adequate pain control on gabapentin, methadone, and highdose oxycodone [56]. While many patients rely on opioids for pain control, the known risks associated with this class of drugs begs for further research into safer treatment options for neuropathic pain.

Another case report describes a 58-year-old patient who suffered a traumatic SCI in Spain; his injury also proved refractory to the aforementioned first-line agents [58]. He tried experimental treatments such as celiac plexus stimulation and neuromodulation to no avail [58]. Moderate results were achieved using hypnosis from a qualified psychiatrist with overall pain levels decreasing from a numeric rating scale (NRS) 7 to an NRS 5 [58]. While this case report illustrates the success of hypnotic intervention in a single patient, it exemplifies the lengths to which individuals will go for even marginal results.

In patients with neuropathic pain secondary to traumatic injury causing central cord syndrome refractory to conventional analgesics, a group of orthopedists in China examined the effect of treatment with methylprednisolone on acute neuropathic pain [59]. In a small sample of 34 patients that was not placebo controlled, patients received seven methylprednisolone infusions daily for 1 week [59]. Allodynia relief exceeding $50 \%$ was seen in $91 \%$ of patients during the 3-month follow-up period [59]. A separate hypothesis included a small, 13-subject, placebo-controlled study that used intrathecal baclofen as an intervention to improve neuropathic pain. Baclofen, a GABA analogue, has been previously shown to exert antinociceptive effects [60]. A single bolus of baclofen was administered after treatment randomization and was observed to result in a significant decrease in neuropathic pain at the 4and 8-h marks, but not the 24-h mark [61]. The baclofen bolus was also associated with a significant decrease in spasticity at the 4-h mark, suggesting that it could be used as an acute intervention [61]. Further, it improved quality of life by decreasing the interference of chronic pain in the daily lives of affected patients [61]. In a French study, the use of ziconotide, an omega-conotoxin analogue that blocks neuronal N-type voltage-sensitive calcium channels, was examined in 20 patients [62]. After an intrathecal injection of ziconotide, 14 of 20 patients had decreases in pain scores greater 
than $40 \%$ [62]. Three of the 14 patients experienced side effects [62]. Two of the patients had serum creatine phosphokinase (CPK) elevations to greater than $3000 \mu \mathrm{g} / \mathrm{L}$ and were immediately withdrawn from treatment; the other patient experienced acute urinary retention and voluntarily withdrew from the study [62]. The remaining 11 of 14 responders opted to have permanent ziconotide pumps implanted [62]. In eight of these 11 patients, the analgesic effect persisted for an average follow-up of over 3 years [62]. The promising effects of methylprednisolone, baclofen, and ziconotide on neuropathic pain should be subject to further exploration.

A Brazilian research group examined two separate areas of the brain as potential targets for central stimulatory therapy [63]. Ninetyeight patients of various ages with clinically diagnosed central neuropathic pain were chosen as subjects for transcranial magnetic stimulation (TMS) of either the ACC or the posterior superior insula (PSI) [63]. These patients underwent repetitive TMS over the course of 16 sessions over 12 weeks [63]. TMS has demonstrated promise in the treatment of fibromyalgia, complex regional pain syndrome, and peripheral neuropathic pain and is believed to act by influencing blood flow and neurotransmitter release [63]. This double-blind study featured a control group receiving placebo stimulation [63]. While pain scores were not significantly different between the two groups, activation of the PSI induced analgesia while activation of the ACC had anxiolytic effects [63]. This observation suggests a proof of concept of a biological response to TMS; the observed significant differences suggest that the framework used in this study provides a method for future research [63].

Transcranial direct current stimulation (tDCS) is an alternative to TMS whose application has also been tested for the reduction of neuropathic pain in patients post-SCI. Thibaut and colleagues assessed the initial and longterm effects of tDCS directed at the primary motor cortex (M1) on pain (visual analogue scale, VAS), quality of life (Patient Health Questionnaire, PHQ-9), and life satisfaction (Satisfaction with Life Scale, SWLS) [55]. M1 was the chosen target given its role in central pain modulation and evidence that M1 stimulation leads to local and distant pain reduction [55]. Patients had the option of enrolling in one or two phases of the randomized controlled study [55]. In the first phase, patients underwent 5 days of tDCS with 3 months of follow-up while in the second phase tDCS was performed for 10 days with 8 weeks of follow-up [55]. Significantly reduced pain was observed at 1 week of follow-up in phase 1 and at 4 weeks in phase 2 [55]. This delay in tDCS effects indicates that the resultant reduction in pain is caused by changes in cortical plasticity rather than immediate changes in excitability [55]. These finding indicate that tDCS, while a promising tool for managing pain in patients with SCI, requires an optimized treatment protocol with repeated stimulation sessions to achieve longlasting reduction in pain [55].

Another potential novel treatment for neuropathic pain utilizes breathing-controlled electrical stimulation (BreEStim) to attempt to dampen autonomic changes that are thought to be associated with neuropathic pain. In short, BreEStim modulates the autonomic system, which is associated with the pain neuromatrix-central autonomic network, an area that has been shown to be partially responsible for mediating pain [64-66]. The successful activation of the pain neuromatrix-central autonomic network can be quantified by looking at combined effects on autonomics as measured by heart rate variability [67]. A small sample of patients post SCI completed a controlled trial in which the treatment group received 120 BreEStim impulses [67]. The treatment group experienced a decrease in heart rate variability, indicating that BreEStim may provide for a viable treatment strategy for decreasing postSCI pain [67].

Individuals with CNP experience major decreases in quality of life secondary to not only pain but also a general decrease in motor and sensory function. Given the well-known importance of physical activity, a Japanese study group looked at a placebo-controlled study of the effect of exercise on neuropathic pain $[68,69]$. Exercise consisted of vigorous wheelchair propulsion while the patient was 
assessed by EEG $[68,69]$. The subject completed a subjective NRS for neuropathic pain and a profile of mood states [68, 69]. Objectively, peak alpha frequencies were measured in four areas of the brain: frontal, central, parietal, and occipital $[68,69]$. In comparison to the control group, the SCI group showed significantly lower pre-wheelchair parietal and occipital peak alpha frequencies that have previously been shown to signify increased neuropathic pain $[68,69]$. Post-exercise central peak alpha frequencies were significantly higher in the SCI group and unchanged in the placebo group [70]. Further, subjective measures of pain were shown to decrease and perceptions of mood were shown to increase following treatment [70].

\section{CONCLUSION}

CNP is a common, chronic complication of SCI that affects a significant proportion of the population; its etiology is attributed to increases in inflammatory mediators and voltage-gated ion channel dysfunction leading to afferent nerve sensitization, chronic pain sensation, and allodynia. This is secondary to increased expression of pro-inflammatory components such as IL- 2 and TNF $\alpha$, and changes in the levels of regulatory microRNA. The underlying mechanism involves the neuron-glia cross talk and activation of the latter. Current treatments include gabapentinoids, TCAs, SNRI, and lidocaine, capsaicin, tramadol, and other opioids if the first group fails to elicit the desired results. Unfortunately, these provide overall limited pain control. More recently, interventional techniques have been studied with mixed results.

The diagnosis of CNP requires a history of a nervous system lesion with a distribution of pain and sensory changes that are in agreement with that lesion location. However, diagnosis is challenging and rarely definitive; many patients also suffer from overlapping chronic pain conditions.

The pathophysiology of CNP most likely begins with nerve injuries leading to local glial and macrophage activation, migration, and proliferation. These inflammatory cells are responsible for the overexpression of pro-inflammatory cytokines that alter the expression milieu of receptors on the cell surface of the glial and neuronal cells; this interaction leads to increased C-fiber signaling and sensitization. Another key player in pathophysiology is the GABAergic neurons; alterations in ion channel expression and activation lead to reduced activation of these neurons, reducing their attenuation capacity.

Treatment options, as listed above, are limited and studies are inconclusive regarding their efficacy, and the data available is limited. Baclofen, an antispastic medication, has been shown to be effective in several studies, possibly linked to the GABAergic theory of pathophysiology; however, more research is required to determine its efficacy. Less conventional treatments, including hypnosis, were tried with some success. More novel drugs are actively being researched; the voltage-sensitive calcium channel blocker ziconotide showed encouraging results, although further research is required to evaluate both efficacy and the toxicity profile.

Non-medical interventions are also being studied, including brain stimulation (TMS, tDCS) and biofeedback techniques (BreEStim), are actively being studied with encouraging preliminary results.

Further research is likely to provide evidence for these and other novel therapeutic agents. A deeper understanding of the molecular events leading to sensitization and chronicity of CNP will lead to the identification of other candidates for therapy. Effective therapy will likely require a multidisciplinary approach that includes lifestyle modifications, psychosocial support, medical and non-medical therapies.

\section{ACKNOWLEDGEMENTS}

Funding. No funding or sponsorship was received for this study or publication of this article.

Authorship. All named authors meet the International Committee of Medical Journal 
Editors (ICMJE) criteria for authorship for this article, take responsibility for the integrity of the work as a whole, and have given their approval for this version to be published.

Disclosures. Omar Viswanath, Ivan Urits, James Burns, Karina Charipova, Kyle Gress, Alexandra McNally, Richard D. Urman, Ali Welschmeyer, Amnon A. Berger, Hisham Kassem, Manuel G. Sanchez, Treniece N. Eubanks, Elyse M. Cornett and Anh L. Ngo have nothing to disclose. Alan D. Kaye is a member of the journal's editorial board.

Compliance with Ethics Guidelines. This article is based on previously conducted studies and does not contain any studies with human participants or animals performed by any of the authors.

Data Availability. Data sharing is not applicable to this article as no datasets were generated or analyzed during the current study.

Open Access. This article is licensed under a Creative Commons Attribution-NonCommercial 4.0 International License, which permits any non-commercial use, sharing, adaptation, distribution and reproduction in any medium or format, as long as you give appropriate credit to the original author(s) and the source, provide a link to the Creative Commons licence, and indicate if changes were made. The images or other third party material in this article are included in the article's Creative Commons licence, unless indicated otherwise in a credit line to the material. If material is not included in the article's Creative Commons licence and your intended use is not permitted by statutory regulation or exceeds the permitted use, you will need to obtain permission directly from the copyright holder. To view a copy of this licence, visit http:// creativecommons.org/licenses/by-nc/4.0/.

\section{REFERENCES}

1. Jensen TS, Baron R, Haanpää M, et al. A new definition of neuropathic pain. Pain. 2011;152:2204-5.

2. Yekkirala AS, Roberson DP, Bean BP, Woolf CJ. Breaking barriers to novel analgesic drug development. Nat Rev Drug Discov. 2017;16:545-64.

3. Alles SRA, Smith PA. Etiology and pharmacology of neuropathic pain. Pharmacol Rev. 2018;70:315-47.

4. Waxman SG, Dib-Hajj S, Cummins TR, Black JA. Sodium channels and pain. Proc Natl Acad Sci USA. 1999;96:7635-9.

5. Abdulla FA, Smith PA. Axotomy- and autotomyinduced changes in $\mathrm{Ca}^{2+}$ and $\mathrm{K}^{+}$channel currents of rat dorsal root ganglion neurons. J Neurophysiol. 2001;84:664-58.

6. Sommer C, Leinders M, Üçeyler N. Inflammation in the pathophysiology of neuropathic pain. Pain. 2018; 159:595-602.

7. Üçeyler N, Rogausch JP, Toyka KV, Sommer C. Differential expression of cytokines in painful and painless neuropathies. Neurology. 2007;69:42-9.

8. Bali KK, Kuner R. Noncoding RNAs: key molecules in understanding and treating pain. Trends Mol Med. 2014;20:437-48.

9. Lehmann SM, Krüger C, Park B, et al. An unconventional role for miRNA: Let-7 activates Toll-like receptor 7 and causes neurodegeneration. Nat Neurosci. 2012;15:827.

10. Park CK, Xu ZZ, Berta T, et al. Extracellular microRNAs activate nociceptor neurons to elicit pain via TLR7 and TRPA1. Neuron. 2014;82:47-544.

11. Zhuang ZY, Kawasaki Y, Tan PH, Wen YR, Huang J, Ji RR. Role of the CX3CR1/p38 MAPK pathway in spinal microglia for the development of neuropathic pain following nerve injury-induced cleavage of fractalkine. Brain Behav Immun. 2007;21: 642-51.

12. Ji RR, Xu ZZ, Gao YJ. Emerging targets in neuroinflammation-driven chronic pain. Nat Rev Drug Discov. 2014;13:533-48.

13. Finnerup NB, Attal N, Haroutounian S, et al. Pharmacotherapy for neuropathic pain in adults: a systematic review and meta-analysis. Lancet Neurol. 2015;14(2):162-73.

14. Warner FM, Cragg JJ, Jutzeler CR, et al. Progression of neuropathic pain after acute spinal cord injury: a 
meta-analysis and framework for clinical trials. J Neurotrauma. 2019;36:1461-8.

15. Finnerup NB, Norrbrink C, Trok K, et al. Phenotypes and predictors of pain following traumatic spinal cord injury: a prospective study. J Pain. 2014;15:40-8.

16. Staudt MD, Clark AJ, Gordon AS, et al. Long-term outcomes in the management of central neuropathic pain syndromes: a prospective observational cohort study. Can J Neurol Sci. 2018;45:545-52.

17. Cepeda MS, Berlin JA, Gao CY, Wiegand F, Wada DR. Placebo response changes depending on the neuropathic pain syndrome: results of a systematic review and meta-analysis. Pain Med. 2012;13: 575-95.

18. Eippert F, Finsterbusch J, Bingel U, Büchel C. Direct evidence for spinal cord involvement in placebo analgesia. Science. 2009;326:404.

19. Vuckovic A, Gallardo VJF, Jarjees M, Fraser M, Purcell M. Prediction of central neuropathic pain in spinal cord injury based on EEG classifier. Clin Neurophysiol. 2018;129:1604-17.

20. Vuckovic A, Jajrees M, Purcell M, Berry H, Fraser M. Electroencephalographic predictors of neuropathic pain in subacute spinal cord injury. J Pain. 2018;19: 1256.

21. Wang Y, Ye F, Huang C, et al. Bioinformatic analysis of potential biomarkers for spinal cord-injured patients with intractable neuropathic pain. Clin J Pain. 2018;34:825.

22. Gruener H, Zeilig G, Laufer Y, Blumen N, Defrin R. Increased psychological distress among individuals with spinal cord injury is associated with central neuropathic pain rather than the injury characteristics. Spinal Cord. 2018;56:176-84.

23. Zhou H, Shi Z, Kang Y, et al. Investigation of candidate long noncoding RNAs and messenger RNAs in the immediate phase of spinal cord injury based on gene expression profiles. Gene. 2018;661: 119-25.

24. Jiang BC, Sun WX, He LN, Cao DL, Zhang ZJ, Gao YJ. Identification of IncRNA expression profile in the spinal cord of mice following spinal nerve ligation-induced neuropathic pain. Mol Pain. 2015;11:43.

25. Zhao X, Tang Z, Zhang H, et al. A long noncoding RNA contributes to neuropathic pain by silencing Kcna2 in primary afferent neurons. Nat Neurosci. 2013;16:1024.
26. Yu HM, Wang Q, Sun WB. Silencing of FKBP51 alleviates the mechanical pain threshold, inhibits DRG inflammatory factors and pain mediators through the NF-kappaB signaling pathway. Gene. 2017;627:196-75.

27. Zhou X, Han X, Wittfeldt A, et al. Long non-coding RNA ANRIL regulates inflammatory responses as a

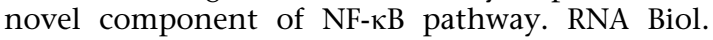
2016;13:98-108.

28. Liu NK, Wang XF, Lu QB, Xu XM. Altered microRNA expression following traumatic spinal cord injury. Exp Neurol. 2009;219:424-9.

29. Ning B, Gao L, Liu RH, Liu Y, Zhang NS, Chen ZY. MicroRNAs in spinal cord injury: potential roles and therapeutic implications. Int $\mathrm{J}$ Biol Sci. 2014;10:997.

30. Liu XJ, Zheng XP, Zhang R, Guo YL, Wang JH. Combinatorial effects of miR-20a and miR-29b on neuronal apoptosis induced by spinal cord injury. Int J Clin Exp Pathol. 2015;8:3811.

31. Widerström-Noga E, Loeser JD, Jensen TS, Finnerup NB. AAPT diagnostic criteria for central neuropathic pain. J Pain. 2017;18:1417-26.

32. Maixner W, Fillingim RB, Williams DA, Smith SB, Slade GD. Overlapping chronic pain conditions: implications for diagnosis and classification. J Pain. 2016;17(9):T93-107.

33. Tang $\mathrm{Y}$, Liu $\mathrm{L}, \mathrm{Xu} \mathrm{D}$, et al. Interaction between astrocytic colony stimulating factor and its receptor on microglia mediates central sensitization and behavioral hypersensitivity in chronic post ischemic pain model. Brain Behav Immun. 2018;68: 248-60.

34. Chhaya SJ, Quiros-Molina D, Tamashiro-Orrego AD, Houlé JD, Detloff MR. Exercise-induced changes to the macrophage response in the dorsal root ganglia prevent neuropathic pain after spinal cord injury. J Neurotrauma. 2019;36:877-90.

35. Boakye PA, Rancic V, Whitlock KH, et al. Receptor dependence of BDNF actions in superficial dorsal horn: relation to central sensitization and actions of macrophage colony stimulating factor 1 . J Neurophysiol. 2019;121(6):2308-22.

36. Du X-J, Chen Y-X, Zheng Z-C, Wang N, Wang X-Y, Kong F-E. Neural stem cell transplantation inhibits glial cell proliferation and $\mathrm{P} 2 \mathrm{X}$ receptor-mediated neuropathic pain in spinal cord injury rats. Neural Regen Res. 2019;14(5):876-85.

37. Lee SH, Shi XQ, Fan A, West B, Zhang J. Targeting macrophage and microglia activation with colony stimulating factor 1 receptor inhibitor is an 
effective strategy to treat injury-triggered neuropathic pain. Mol Pain. 2018;14:1-12.

38. Kusuyama K, Tachibana T, Yamanaka H, Okubo M, Yoshiya S, Noguchi K. Upregulation of calcium channel alpha-2-delta-1 subunit in dorsal horn contributes to spinal cord injury-induced tactile allodynia. Spinal J. 2018;18(6):1062-9.

39. Sánchez-Brualla I, Boulenguez P, Brocard C, et al. Activation of 5-HT 2A receptors restores KCC2 function and reduces neuropathic pain after spinal cord injury. Neuroscience. 2018;387:48-57.

40. Li T, Wan Y, Sun L, et al. Inhibition of microRNA$15 \mathrm{a} / 16$ expression alleviates neuropathic pain development through upregulation of $G$ proteincoupled receptor kinase 2. Biomol Ther. 2019;27(4): 414-22.

41. Yang $\mathrm{Z}, \mathrm{Xu} \mathrm{J}, \mathrm{Zhu} \mathrm{R}$, Liu L. Down-regulation of miRNA-128 contributes to neuropathic pain following spinal cord injury via activation of P38. Med Sci Monit. 2017;23:405-11.

42. Miranpuri GS, Schomberg DT, Alrfaei B, et al. Role of matrix metalloproteinases 2 in spinal cord injury-induced neuropathic pain. Ann Neurosci. 2016;23(1):25-322.

43. Gui Y, Duan S, Xiao L, Tang J, Li A. Bexarotent attenuated CCI-induced spinal neuroinflammation and neuropathic pain by targeting MKP-1. J Pain. 2019. https://doi.org/10.1016/j.jpain.2019.01.007.

44. Castany S, Gris G, Vela JM, Verdú E, Boadas-Vaello P. Critical role of sigma-1 receptors in central neuropathic pain-related behaviours after mild spinal cord injury in mice. Sci Rep. 2018;8(1):1-3.

45. Sanna MD, Les F, Lopez V, Galeotti N. Lavender (Lavandula angustifolia Mill.) essential oil alleviates neuropathic pain in mice with spared nerve injury. Front Pharmacol. 2019;10:472.

46. Liu Z, Song Z, Guo S, et al. CXCL12/CXCR4 signaling contributes to neuropathic pain via central sensitization mechanisms in a rat spinal nerve ligation model. CNS Neurosci Ther. 2019;25(9): 922-36.

47. Wu XB, He LN, Jiang BC, et al. Spinal CXCL9 and CXCL11 are not involved in neuropathic pain despite an upregulation in the spinal cord following spinal nerve injury. Mol Pain. 2018. https://doi.org/ $10.1177 / 1744806918777401$.

48. Ellis A, Grace PM, Wieseler J, et al. Morphine amplifies mechanical allodynia via TLR4 in a rat model of spinal cord injury. Brain Behav Immun. 2016;58:348-56.
49. Vicario N, Pasquinucci L, Spitale FM, et al. Simultaneous activation of $\mathrm{mu}$ and delta opioid receptors reduces allodynia and astrocytic connexin 43 in an animal model of neuropathic pain. Mol Neurobiol. 2019;56(11):7338-544.

50. Warner FM, Cragg JJ, Jutzeler CR, et al. The progression of neuropathic pain after acute spinal cord injury: a meta-analysis and framework for clinical trials. J Neurotrauma. 2018;36(9):1461-8.

51. Huang W, Kabbani N, Brannan T, et al. Association of a functional polymorphism in the CHRFAM7A gene with inflammatory response mediators and neuropathic pain after spinal cord injury. J Neurotrauma. 2018;36(21):3026-33.

52. Wang $\mathrm{A}, \mathrm{Xu} \mathrm{C}$. The role of connexin 43 in neuropathic pain induced by spinal cord injury. Acta Biochim Biophys Sin. 2019;51(6):555-61.

53. Tonkin R, Bowles C, Perera C, Keating B, Makker P, Duffy S. Attenuation of mechanical pain hypersensitivity by treatment with peptide5, a connexin43 mimetic peptide, involves inhibition of NLRP3 inflammasome in nerve-injured mice. Exp Neurol. 2018;300:1-12.

54. Rosendahl A, Krogh S, Kasch H. Pain assessment in hospitalized spinal cord injured patients-a controlled cross-sectional study. Scand J Pain. 2018;19(2):299-307.

55. Thibaut A, Carvalho S, Morse L, Zafonte R, Fregni F. Delayed pain decrease following M1 tDCS in spinal cord injury: a randomized controlled clinical trial. Neurosci Lett. 2017;658:19-26.

56. Horan NA, Pugh TM. Intractable central pain in a patient with diffuse glioma. Am J Phys Med Rehabil. 2019;98:107-10.

57. Song K-S, Cho JH, Hong J-Y, et al. Neuropathic pain related with spinal disorders: a systematic review. Asian Spine J. 2017;11(4):661-74.

58. Daszkiewicz A, Gierlotka Z, Nierodzinski W, Misiolek A, Misiolek H. Neuropathic pain after spinal cord injury resistant to conventional therapies-case report. Psychatr Pol. 2016;50(2):345-55.

59. Li L, Han Y, Li T, Zhou J, Sun C, Xue Y. The analgesic effect of intravenous methylprednisolone on acute neuropathic pain with allodynia due to central cord syndrome: a retrospective study. J Pain Res. 2018;11(1231-1238):1231-8.

60. Hammond D, Drower E. Effects of intrathecally administered THIP, a baclofen and muscimol on nociceptive threshold. Eur J Pharmacol. 1984;103: 121-5. 
61. Kumru H, Benito-Penalva J, Kofler M, Vidal J. Analgesic effect of intrathecal baclofen bolus on neuropathic pian in spinal cord injury patients. Brain Res Bull. 2018;140:205-11.

62. Brinzeu A, Berthiller J, Caillet J-B, Staquet H, Mertens P. Ziconotide for spinal cord injury-related pain. Eur J Pain. 2019;23(9):1688-700.

63. Galhardoni R, da Silva VA, Garcia-Larrea L, et al. Insular and anterior cingulate cortex deep stimulation for central neuropathic pain: disassembling the percept of pain. Neurology. 2019;92(18):2165-75.

64. Melzack R. Pain and neuromatrix in the brain. J Dent Educ. 2001;65(12):1378-82.

65. Melzack R, Wall P. Pain mechanisms: a new theory. Surv Anesth. 1967;11:89-90.

66. Melzack R, Loeser J. Phantom body pain in paraplegics: evidence for a central "pattern generating mechanism" for pain. Pain. 1977;4:195-21010.
67. Karri J, Li S, Zhang L, Chen Y-T, Stampas A, Li S. Neuropathic pain modulation after spinal cord injury by breathing-controlled electrical stimulation (BreEStim) is associated with restoration of autonomic dysfunction. J Pain Res. 2018;11: 2331-41.

68. Boord P, Siddall P, Tran Y, Herbert D, Middleton J, Craig A. Electroencephalographic slowing and reduced reactivity in neuropathic pain following spinal cord injury. Spinal Cord. 2008;46:118-23.

69. Wydenkeller S, Maurizio S, Dietz V, Halder P. Neuropathic pain in spinal cord injury: significance of clinical and electrophysiological measures. Eur J Neurosci. 2009;30:91-9.

70. Sato G, Osumi M, Morioka S. Effects of wheelchair propulsion on neuropathic pain and resting electroencephalography after spinal cord injury. J Rehabil Med. 2017;49:136-43. 